\title{
A classic game in modern time inside educational context
}

\author{
Leonardo Saucedo, Karina Figueroa, Cuauhtémoc Rivera-Loaiza
}

Published: 30 November 2020

\begin{abstract}
Many educational strategies based on using games have been part of the teacher toolset for a long time. Within the teaching profession in Mexico it is a common practice to use traditional games and use them as part of the academic materials created by the teachers themselves (Mexican lottery, bingo, cards, etc.), in order to boost the engagement of the students into the learning process. Also, usage of smartphones by students has increased significantly, and therefore the potential of allowing educators to design their own digital games to teach something in particular. In this article we show the design, creation and personalization of a classical board game known as "memorama" to be used in a digital manner. The main idea is that a user (i.e. teacher) will define pairs of cards (question and answer) and these will form the deck of digital cards of the game. This game has potentially a high impact because it is customizable nature. This work was partially presented in XII Congreso de la Mujer en la Ciencia in poster track.
\end{abstract}

\section{Keywords}

Gamification; User-Centered Design; Usability; Education tools.

\section{Introducción}

Uno de los problemas fundamentales en la educación básica en la actualidad es el lograr que los estudiantes puedan concentrarse adecuadamente en la obtención del conocimiento que deben de adquirir en su proceso educativo. La atención de los alumnos es continuamente requerida por el profesor, sus compañeros y todo el ecosistema tecnológico actual. Teléfonos inteligentes, tabletas electrónicas, equipo de cómputo, consolas de videojuegos y dispositivos similares son parte de los elementos con los que los estudiantes conviven todo el tiempo y los que en muchos casos son el componente principal para interactuar con su entorno inmediato (digital o analógico). Es precisamente ese uno de los principales retos en la educación hoy en día: ¿de qué manera es posible integrar todos los elementos mencionados dentro de una estrategia que promueva un aprendizaje eficiente?

Saucedo, L.., Figueroa, K.., Rivera-Loaiza, C.

Universidad Michoacana de San Nicolás de Hidalgo

ldsaucedo@gmail.com, karina.figueroa@umich.mx,

cuauhtemoc.rivera@umich.mx
Un área que muestra un avance promisorio es la gamificación dentro del proceso educativo. La gamificación (también conocida como ludificación) se refiere a la utilización de estrategias clásicas de juego para lograr un objetivo de capacitación o enseñanza. Una de las ventajas de este enfoque es el incrementar la motivación de los estudiantes mediante un ambiente competitivo bajo la supervisión de el maestro.

En particular, el juego de memorama o juego de memoria comienza con un conjunto de cartas por un lado con información (imágenes o texto) y por otro un color sólido o patrón (todas tienen el mismo diseño en este lado), que deben revolverse y repartirse sobre una "mesa" con la información hacia abajo, de tal suerte que no sea posible ver la información que se encuentra en ellas. De manera inicial, un jugador puede ver todas las cartas exactamente iguales.

El juego consiste en que un jugador, selecciona 2 cartas y las voltea para ver su contenido, si ambas cartas corresponden, entonces ese par se retira de las cartas restantes y se quedan con el jugador que las descubrió. Además tiene derecho a seleccionar otras 2 cartas. Si las nuevas cartas son diferentes, entonces deberá ceder el turno a otro jugador.

El siguiente jugador debe seleccionar 2 cartas (pueden ya haber sido destapadas). Este jugador tiene una ventaja respecto al primero, ya debió haber visto las cartas que otro(s) jugador(es) ha (n) destapado anteriormente y recordar las posiciones de esas cartas. El juego termina cuando todos los pares son destapados, por supuesto, gana aquel jugador que logró conseguir más pares correctos.

La idea de este juego es un ejercicio de memoria, y en nuestro caso, nuestro objetivo es aprovechar este momento para hacer un reforzamiento del contenido académico.

Este artículo está organizado de la siguiente manera: en la sección 2 se presenta la metodología empleada en el diseño, la base de datos, el desarrollo y la implementación multijugador. Después en la sección 3 se presentan los resultados del desempeño de la aplicación con un grupo de prueba. Finalmente en la sección 4 se presentan las conclusiones y trabajo a futuro.

\section{Metodología}

Para nuestra propuesta decidimos emplear el proceso de desarrollo denominado Diseño Centrado en el Usuario. Conocido mejor por sus siglas en inglés, User-Centered Design (UCD) es una metodología que describe los procesos de diseño que los usuarios finales influyen en cómo un diseño toma forma [9]. Es una filosofía de diseño que tiene por objetivo la creación de productos que resuelvan necesidades concretas de sus usuarios.

La metodología empleada para el diseño y creación de este juego se llevó a cabo de la siguiente forma, primero se revisamos distintas aplicaciones y tipos de juegos que pudieran ser personalizados y cuya curva de aprendizaje fuera mínima. Una vez 
que se decidió por un juego muy clásico como lo es el memorama, se diseñó lo que podría ser la interfaz del juego y la ruta que podrían seguir los usuarios (diagrama de uso). Finalmente se implementó como una app para teléfono móvil con sistema operativo Android.

\subsection{Diseño}

Para nuestro juego, un par de cartas corresponde a una preguntarespuesta académica, por ejemplo, $2+2$ podría ser una pregunta y 4 la respuesta. Note que casi cualquier contenido académico puede ser expresado de esta forma. Por otro lado, dado que el juego será personalizable, los usuarios podrán decidir el número de preguntas que desee poner en cada juego.

\subsubsection{Usuarios}

Cabe mencionar que la aplicación cuenta con los distintos personajes: un diseñador de un juego, un jugador. Ambos personajes pueden jugar o diseñar un juego por lo que simplemente diremos que cada usuario podrá realizar ambas acciones.

El diseño de las interfaces de esta aplicación está organizada por las siguientes categorías: entrada de datos, consulta de información y juego.

\subsubsection{Interfaces para entrada de datos}

Dado que es una aplicación personalizable se requiere identificar al usuario que está manejando la aplicación, para esto se diseñó una interfaz para el registro de nuevos usuarios y registro de usuarios existentes.

- Para esta interfaz es necesario tener 3 opciones: entrar (usuarios previamente registrados), registrarse y recordar la contraseña. Tabla, lado izquierdo.

- Interfaz para la captura de cada par (pregunta-respuesta), véase la Figura 1-derecha. Cabe mencionar que cada juego debe tener un título asignado por el usuario y una descripción, así como un número de juego asignado por la plataforma
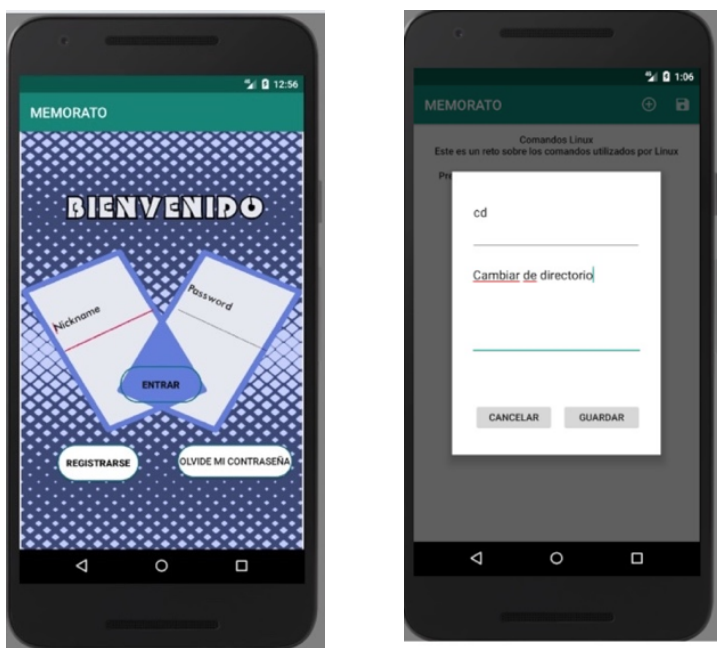

Figura 1. Interfaces de captura de datos. En la izquierda se tiene la interfaz de inicio, mientras que en la derecha se tiene la interfaz de captura de un par.

\subsubsection{Interfaces de información}

En el caso de la información que el usuario requiere conocer son: juegos creados, juegos jugados, estadísticas sobre juegos diseñados, y en un juego en particular se requiere conocer todos los pares capturados.
En la Figura 2 (izquierda) se muestra una pantalla con la lista de pares (pregunta-respuesta) para un juego cuyo título es: Comandos de Linux. La descripción es un mensaje breve sobre lo que un jugador puede esperar de este juego. Después viene una lista de preguntas capturadas por el usuario. Como se puede apreciar existe una línea que separa cada pregunta. El primer renglón es la pregunta y el segundo renglón (el que empieza con R) corresponde a la respuesta.

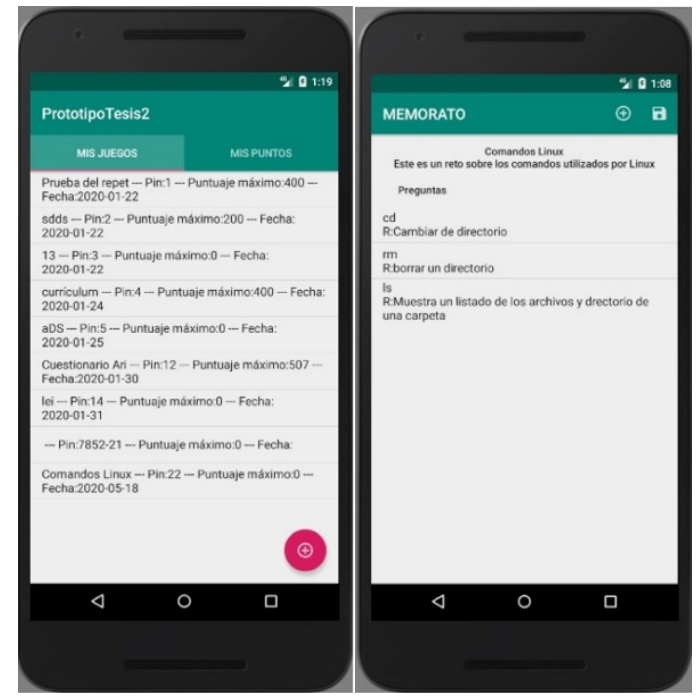

Figura 2. Interfaz de información. En el lado izquierdo se muestra una la lista con 3 preguntas capturadas por un usuario para un juego titulado Comandos de Linux.

\subsubsection{Interfaces de juego}

Una vez definidas las preguntas para un juego se presentará la siguiente interfaz (véase la Figura 3). Inicialmente todas las cartas están ocultas, y bastará darle clic a alguna de las cartas para que se muestre al centro de la pantalla con la información visible, después de un breve instante regresará a la posición inicial destapada, hasta que se destape una segunda y transcurra unos segundos.

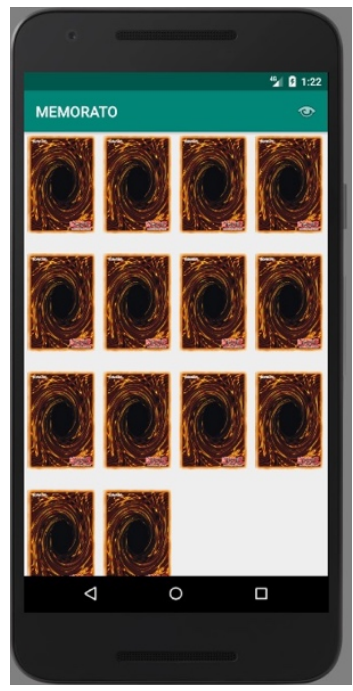

Figura 3. Juego de memorama con 7 pares por descubrir. 


\subsection{Base de Datos}

Para llevar acabo esta aplicación se requería un sistema de base de datos capaz de almacenar la información emitida por este juego. Todo esto con base en los principios descritos en [7]. Básicamente hay 3 entidades: persona, juego y pregunta. Las relaciones son: persona-juega-juego, persona-crea-juego, juego-tiene-preguntas. Véase la Figura 4 para mas detalles.

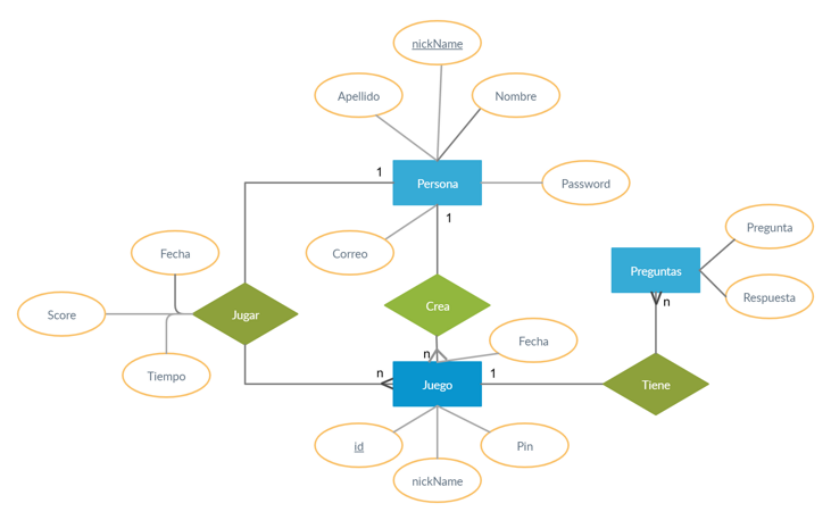

Figura 4. Diagrama de base de datos relacional.

\subsection{Diagrama de Uso}

Por otro lado, también se diseñó un diagrama de navegación de este juego, de acuerdo a [8] éste es presentado en la Figura 5. El diagrama solo representa el flujo de pantallas que se presenta a cada usuario con sus respectivas opciones.

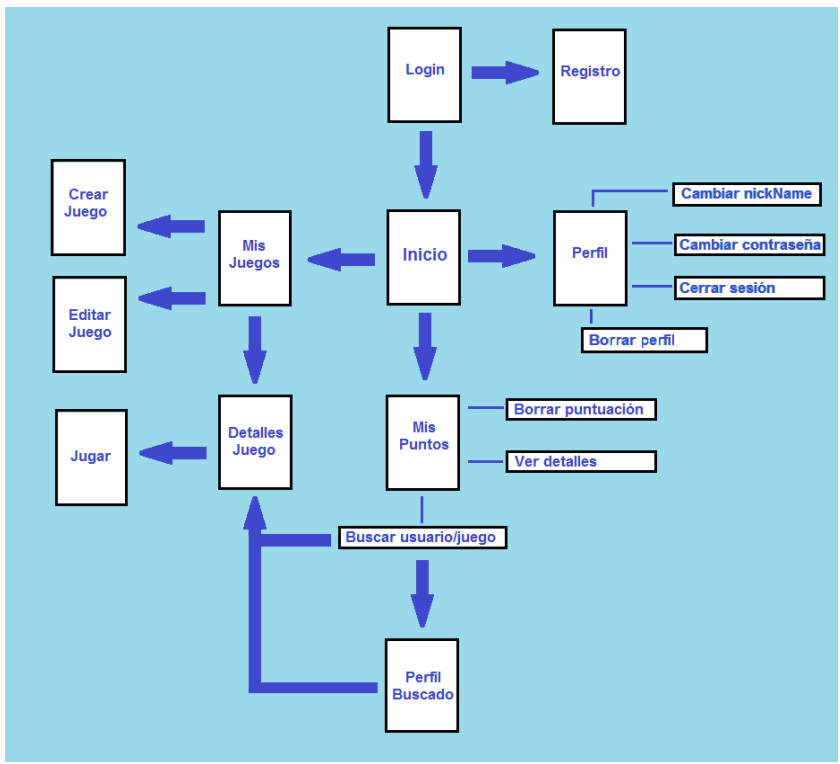

Figura 5. Diagrama de navegación del juego.

\subsection{Desarrollo}

Las herramientas tecnológicas utilizadas fueron:

- Plataforma de desarrollo para la codificación de la aplicación se utilizó: Android studio [3,4].

- Para almacenar la base de datos se usó un sistema gratuito llamado firebase $[5,6]$.

\subsection{Modo Multijugador}

Esta aplicación está diseñada para que se pueda jugar en línea entre varios jugadores. La función de la aplicación será controlar quien tiene el turno de jugar, para esto, se bloquea la pantalla de un jugador para evitar que pueda destapar casos cuando no le corresponde.

\section{Evaluación de la aplicación}

La aplicación actualmente ya se encuentra disponible en la plataforma de google play. Para llevar a cabo la evaluación de la aplicación se solicitó la colaboración de un grupo de profesores en conjunto con sus estudiantes de un instituto de nivel medio superior. Los temas de las materias fueron: biología, español y química.

\subsection{Profesores}

Para explicar la evaluación de la aplicación primero se abordará el trabajo realizado para los profesores. Básicamente consistió en 3 partes: evaluación de sus habilidades TIC, uso de la plataforma y finalmente se les pidió realizar un cuestionario para evaluar el conocimiento de sus estudiantes antes y después de utilizar la aplicación

\subsubsection{Evaluación de habilidades TIC a profesores}

Para llevar a cabo la evaluación de los docentes primero se les aplicó un cuestionario para conocer su grado de familiaridad con las TIC. Las preguntas realizadas fueron:

- $\quad$ Edad

- ¿Utiliza alguna herramienta tecnológica en sus clases?

- ¿Cómo usa las TIC en clase?

- ¿Conoce alguna app para teléfono que le sirva como herramienta para reforzar los conocimientos vistos en clase?

- ¿Le gustaría utilizar una app lúdica para reforzar los temas vistos en clase?

La edad de los docentes estuvo entre los 25 y 35 años. En la encuesta aplicada sus respuestas fueron en el sentido de saber utilizar las TIC, sin embargo, no las involucran en sus clases pues no siempre pueden encontrar la aplicación/página/video acorde al material que verán en cada clase. Esto aunado al tiempo involucrado en la búsqueda del material correcto para el tema tratado en clase, además de la curva de aprendizaje que se necesita para cada nueva herramienta que pudieran encontrar.

\subsubsection{Uso de la aplicación}

A los profesores que aceptaron ser parte del experimento se les dio una breve explicación de la aplicación y se dejó que ellos la usaran.

La tarea específica para ellos fue que crearan un juego con un conjunto de preguntas que ya tenían creadas previamente.

El resultado fue satisfactorio, todos los profesores consiguieron la tarea rápidamente.

\subsection{Alumnos}

Para el caso de los alumnos de los profesores involucrados, se seleccionaron alumnos de dos grupos donde los profesores daban clases bajo ciertas características las cuales fueron: alumnos que aprendieran rápido, alumnos que tuvieran cierta dificultad para aprender pero estudiaran mucho, alumnos que a pesar de hacer un gran esfuerzo se les dificultara aprender y alumnos que tuvieran problemas de aprendizaje y no hiciera un esfuerzo extra escolar para aprender, teniendo un total de 9 alumnos. 
El proceso seguido con los alumnos consistió en: evaluación de conocimientos previos sobre los temas de interés (uno por cada materia), posteriormente se les evaluó sus habilidades TIC específicamente en un teléfono móvil.

\subsubsection{Evaluación de conocimientos a los estudiantes}

Un tema muy importante antes de evaluar si la aplicación consigue mejorar el aprovechamiento escolar es saber cual es el grado de conocimiento de los estudiantes por lo que se les aplicó un cuestionario de cada tema (biología, español y química). La Figura 6 muestra las calificaciones obtenidas en cada una de las materias de interés.

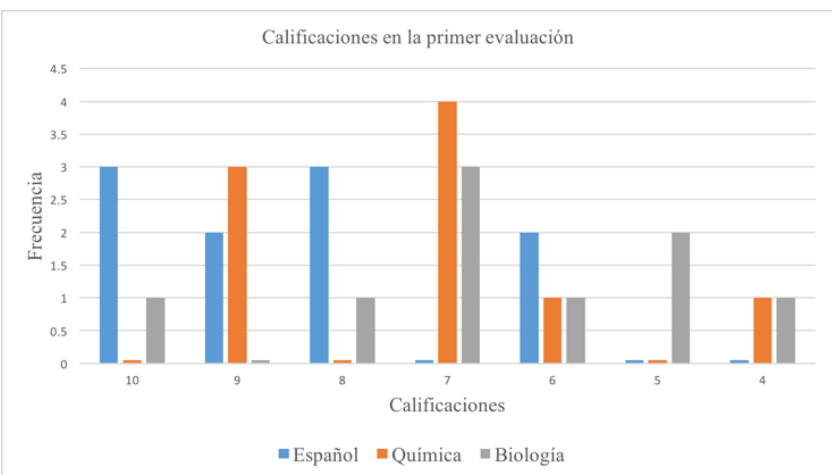

Figura 6. Resultados de la primera evaluación en español, química y biología.

\subsubsection{Evaluación de habilidades TIC}

De los jóvenes participantes nos interesaba saber su grado de involucramiento con las TIC. A este grupo de jóvenes se les preguntó lo siguiente:

- Edad

- ¿Usas de manera regular tu celular?

- ¿Cuál es tu experiencia de aplicaciones para repasar una lección vista en clase?

- ¿Tus profesores usan este tipo de herramientas para reforzar tu aprendizaje?

La edad de estos jóvenes oscila entre 18 y 20 años. La cantidad de tiempo que emplean estos jóvenes varía entre 8 y 12 horas al día. Entre las aplicaciones más utilizadas están Facebook, WhatsApp y YouTube.

Un dato interesante fue que los jóvenes utilizan una aplicación llamada Duolingo para su aprender idiomas

Otros datos interesantes fueron que a los estudiantes les parece muy interesante y motivador la plataforma de Kahoot que algunas veces utilizan sus profesores, además mencionaron también a Geogebra para el entendimiento de matemáticas.

\subsubsection{Uso de la aplicación}

La evaluación de la aplicación se llevó acabo de la siguiente manera:

- Se explicó brevemente en qué consiste un memorama

- Se les instaló en su teléfono celular

- Se les pidió que se registraran, hasta aquí es un paso muy clásico en cualquier aplicación

- Se les proporcionó el id del juego que habían preparado sus profesores

Una vez que los usuarios jugaron varias veces cada juego, se les explicó la opción multijugador, lo cual incrementó su nivel de interés.
Después de hora y media que estuvieron jugando se detuvo la interacción con el juego para pasar a la siguiente etapa, la evaluación de conocimientos nuevamente.

\subsubsection{Evaluación de conocimientos después del uso de la aplicación}

Después del uso de la aplicación se volvió a aplicar el mismo cuestionario aplicado a priori que se refiere en la sección 3.2.1. Los resultados fueron gratamente mucho mejores. Como se muestra en la Figura 7.

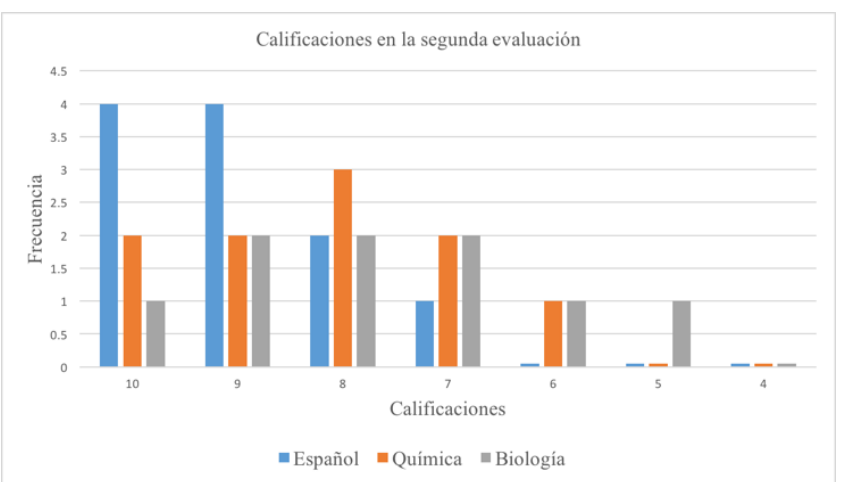

Figura 7. Resultados de la 2a evaluación en español, química y biología.

De la Figura 7 (2a evaluación, barras de color azul) se puede ver que los estudiantes tuvieron un mejor aprendizaje de los mismos temas. La gráfica previa se muestra en la Figura 6 (barras azules).

Para el caso de Química también se nota una mejora considerable. Nótese que en la Figura 6 (barras naranjas) se nota una dispersión de las calificaciones entre 9 y 4, sin embargo, en la Figura 7 (barras naranjas) se puede apreciar como los resultados están más concentrados entre el rango de calificación de 10 y 6 .

Para el caso de Biología, en la comparación se hace con respecto a las Figuras 6 y 7 en las barras color gris. Nótese que los valores antes dispersos entre 8 y 4 , después de la aplicación las calificaciones están más concentradas en el rango entre 9 y 5 .

\section{Conclusiones}

El uso de juegos para captar la atención de los estudiantes y orientarlo hacia el refuerzo de su aprendizaje no es un tema nuevo, sin embargo, y a pesar de la gran cantidad de herramientas que existen, muy pocas de ellas son personalizables por un profesor. En este artículo se presentó el diseño, desarrollo y creación de un juego clásico a uno digital: el memorama.

El juego del memorama consiste en tener un conjunto de pares ocultos en cartas, en cada jugada, es posible destapar de todas las cartas y si ambas corresponden al par, entonces dichas cartas se remueven de las ocultas. Sino coincidieran deberán voltearse y dejarse en el mismo lugar. El juego termina cuando todas las cartas han encontrado su par correspondiente. Las reglas de juego se enfocaron a tener un par, pregunta-respuesta de cualquier tema, iy a jugarse! La idea general es que un alumno reforzará su aprendizaje mientras destapa una carta y debe estar pensando en su complemento.

En este artículo se presentó el diseño, adaptación y desarrollo de un juego clásico: el memorama. Además, se muestra el desempeño de dicho juego aplicado a 10 alumnos de nivel medio superior que colaboraron para analizar el uso de la aplicación. 
Este juego propuesto podría coadyuvar a la memorización de material con el que los estudiantes tienen dificultad. El modelo planteado en este artículo para adaptar un juego clásico en esta época moderna fue utilizando la tecnología existente: software para el desarrollo de aplicaciones móviles, una base de datos en la nube como firebase y el cambio de una pareja de elementos iguales a una pareja formada por pregunta-respuesta.

Como trabajo futuro planeamos hacer una prueba de un grupo de usuarios más grande y con la opción de multijugador.

\section{Referencias}

[1] Breen, D. (2017). Diseña juegos digitales. EUA: NA.

[2] D. Vallejo, e. a. (2017). Desarrollo de Videojuegos un enfoque práctico. Castilla, España.

[3] Hebuterne, S. (2018). Desarrolle una aplicación android. Programación en Java con android studio . España: Eni.

[4] Luján Castillo, J. D. (2017). Android Studio - Aprende a desarrollar aplicaciones. España: Alfaomega, RC Libros.
[5] Moroney, L. (2017). The definitive guide to firebase. Washington: Apress.

[6] S, A. K. (2018). Mastering Firebase for Android Development: Build real-time, scalable, and cloud-enabled Android apps with Firebase. Birmingham: Packt.

[7] Silberschatz, A., Korth, H., \& Sudarshan, S. (2014). Fundamentos de bases de datos (Vol. 6). España: Mcgraw Hill.

[8] Wood, D. (2015). Diseño de Interfaces. España: Parramon.

[9] Cuauhtémoc Rivera-Loaiza, María Isabel López-Huerta, and Francisco J. Domínguez-Mota. 2019. Use of augmented reality to teach basic computing concepts. In Proceedings of the IX Latin American Conference on Human Computer Interaction (CLIHC '19). Association for Computing Machinery, New York, NY, USA, Article 20, 1-6. DOI:https://doi.org/10.1145/3358961.3358981

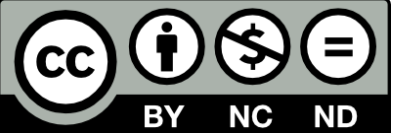

(C) 2020 by the authors. This work is licensed under the Creative Commons AttributionNonCommercial-NoDerivatives 4.0 International License. To view a copy of this license, visit http://creativecommons.org/licenses/by-nc-nd/4.0/ or send a letter to Creative Commons, PO Box 1866, Mountain View, CA 94042, USA. 